\title{
Fuzzy Rankings for Preferences Modeling in Group Decision Making
}

\author{
Nicola Capuano \\ Dep. of Information, Electric Engineering and Applied Mathematics, University of Salerno \\ Via Giovanni Paolo II 132, 84084 Fisciano (SA), Italy. (e-mail: ncapuano@unisa.it) \\ Francisco Chiclana \\ School of Computer Science and Informatics, Faculty of Technology, De Montfort University \\ Leicester LE1 9BH, UK (e-mail: chiclana@dmu.ac.uk) \\ Enrique Herrera-Viedma ${ }^{1}$ \\ Dep. of Computer Science and Artificial Intelligence, University of Granada \\ Granada 18071, Spain (e-mail: viedma@decsai.ugr.es) \\ Hamido Fujita \\ Faculty of Software and Information Science, Iwate Prefectural University \\ Iwate, Japan (e-mail: HFujita-799@acm.org) \\ Vincenzo Loia \\ Dep. of Management and Innovation Systems, University of Salerno \\ Via Giovanni Paolo II 132, 84084 Fisciano (SA), Italy (e-mail: loia@unisa.it)
}

\begin{abstract}
Although Fuzzy Preference Relations (FPRs) are among the most commonly used preference models in Group Decision Making (GDM), they are not free from drawbacks. First of all, especially when dealing with many alternatives, the definition of FPRs becomes complex and time-consuming. Moreover they allow to focus on only two options at a time. This facilitates the expression of preferences but let experts lose the global perception of the problem with the risk of introducing inconsistencies that impact negatively on the whole decision process. For these reasons, different preference models are often adopted in real GDM settings and, if necessary, transformation functions are applied to obtain equivalent FPRs. In this paper we propose Fuzzy Rankings, a new approximate preference model that offers an higher level of user-friendliness with respect to FPRs while trying to maintain an adequate level of expressiveness. Fuzzy rankings allow experts to focus on two alternatives at a time without losing the global picture so reducing inconsistencies. Conversion algorithms from fuzzy rankings to FPRs and backward are defined as well as similarity measures, useful when evaluating the concordance between experts' opinion. A comparison of the proposed model with related works is reported as well as several explicative examples.
\end{abstract}

Keywords: Group Decision Making; Fuzzy Preference Relations; Fuzzy Rankings.

\section{Introduction and Related Work}

Group Decision Making (GDM) is a problem-solving activity by which a group of experts collectively select a belief or a course of action among several available alternatives. A GDM process consists in the evaluation of the alternatives and the choice of the most satisfactory one, taking into account all the

\footnotetext{
${ }^{1}$ Corresponding author
} 
factors and contradictory requirements and according to the preferences of the majority of involved experts. GDM has been widely studied since it has applications in many fields and several approaches have been proposed so far for the representation of experts' preferences, for their aggregation, for the selection of the best alternative and for consensus reaching $[1,2,3,4,5,6]$.

By looking at the representation of experts' preferences (that is the focus of this paper) we see that many preference models already exist. With ordinal rankings [7] experts are asked to order the alternatives from the best to the worst; with utility vectors [8] they must assign an utility value to each alternative; with fuzzy estimates [9] they must assign them a linguistic evaluation that is subsequently translated into a fuzzy number; with preference relations [10] they must select, for every pair of alternatives, the preferred one; with Fuzzy Preference Relations (FPRs) [11] they must assign a degree of preference for each alternative over any other.

Among existing models, ordinal ranking is the most simple and user-friendly and its adoption is especially useful when experts have difficulties in assessing quantitatively the strength of their preferences. For this reason, according to [12], using ordinal rankings reduces the possibility of deriving recommendations based on incorrect information. Conversely, the most diffused preference model is FPR. According to $[13,14]$, it allows experts to focus on two alternatives at a time facilitating the expression of more accurate preferences with respect to non-pairwise methods. It also ensures a high level of expressiveness and translation techniques are available to convert preference information from many other models to FPR [3].

Although ordinal rankings are user-friendly and help reducing errors and inconsistencies, they are considered too simplistic to model preferences in real GDM settings. Experts in fact can be unable to assign a precise position in a ranking to alternatives that are considered equivalent or they may need to specify at what extent an alternative is better than the next preferred one. On the other hand, FPRs definition is often too complex and time-consuming. Moreover, they impose unnecessary precision in preference definition facilitating the emergence of errors and inconsistencies that impact the whole GDM process.

To overcome these limitations, we introduce in this paper a new preference model named Fuzzy Ranking that can be considered a fair compromise between the expressive capability of FRP and the userfriendliness of ordinal ranking. Like for ordinal rankings, experts are asked to order alternatives from the best to the worst but, using specific separators, experts can extend or contract the gap between subsequent positions to reinforce or weaken the ordering relation. Ties are admitted as well as partial and multiple rankings: partial rankings are useful when an expert is unable to evaluate some alternatives; multiple rankings helps an expert state that some alternatives are incomparable with some others.

A first formulation of fuzzy ranking has been proposed in [15] for the expression of ordinal assessment statements within a work aimed at the application of GDM techniques in peer grading. The same model has been also adopted in [16] to collect expert preferences within a GDM model guided by social influence. In this paper we formalize and extend the model outlined in such papers, define conversion functions and similarity measures and compare the model with related work.

The paper is organized as follows: section 2 provides background concepts related to GDM, useful to appreciate the subsequent sections; section 3 defines the fuzzy ranking model; sections 4 and 5 present conversion algorithms to and from FPRs; section 6 introduces partial and multiple fuzzy rankings; section 7 defines similarity measures on fuzzy rankings; section 8 presents similarities and differences with related work; section 9 points out conclusions.

\section{Background Concepts on GDM}

A GDM problem is characterized by a group of experts $E=\left\{e_{1}, \ldots, e_{m}\right\}$, each with her own knowledge, ideas, experience and motivation, that express their preferences on a finite set of alternatives $X=$ $\left\{x_{1}, \ldots, x_{n}\right\}$ to achieve a common solution $[17,18,19]$. As anticipated in section 1 , several ways to express and model experts' preferences have been proposed. Among them, we introduce below the most closely related to our research: ordinal ranking and FPR. 
Ordinal ranking is one of the simplest preference models. An ordinal ranking on elements of $X$ can be denoted as: $x_{\sigma(1)}>\cdots>x_{\sigma(n)}$ where $\sigma:\{1, \ldots n\} \rightarrow\{1, \ldots n\}$ is a permutation function. It can be alternatively represented through an ordering array $O=\left(o_{1}, \ldots, o_{n}\right)$ where each element $o_{i} \in\{1, \ldots, n\}$ states the position of $i$-th alternative within the ranking.

$F P R$ is one of the most commonly used preference models [20, 21, 22]. It specifies the degree to which each alternative $x_{i} \in X$ is at least as good as any other alternative $x_{j}$ by means of a fuzzy relation $P$ i.e. a fuzzy set on $X \times X$ with a membership function $\mu_{P}: X \times X \rightarrow[0,1]$ such that [23]:

$$
\mu_{P}\left(x_{i}, x_{j}\right)= \begin{cases}1 & \text { if } x_{i} \text { is definitely preferred to } x_{j} \\ a \in(0.5,1) & \text { if } x_{i} \text { is slightly preferred to } x_{j} \\ 0.5 & \text { if } x_{i} \text { and } x_{j} \text { are evenly preferred, } \\ b \in(0,0.5) & \text { if } x_{j} \text { is slightly preferred to } x_{i} \\ 0 & \text { if } x_{j} \text { is definitely preferred to } x_{i} .\end{cases}
$$

A FPR can be conveniently represented as a $n \times n$ matrix $P=\left(p_{i j}\right)$ where $p_{i j}=\mu_{P}\left(x_{i}, x_{j}\right)$. A FPR satisfying the additive reciprocity property so that $p_{i j}+p_{j i}=1 \forall i, j \in\{1, \ldots, n\}$, is said to be reciprocal. A FPR that satisfies the additive transitivity property so that $p_{i j}+p_{j k}+p_{k i}=1.5 \forall i, j, k \in$ $\{1, \ldots, n\}$, is also said to be additive consistent.

Sometimes, due to domain complexity, limited expertise or pressure to make a decision, experts define incomplete FPRs [24, 25]. In [26, 27], a method to estimate missing FPR values applying reciprocity and additive transitivity properties is proposed while in [28,29] alternative methods are introduced based on different properties. Using the definition of additive transitivity, it is possible to obtain the following estimates of any missing preference $p_{i j}$, of alternative $x_{i}$ over $x_{j}$, using an intermediate alternative $x_{k}$ with $x_{i}, x_{j}, x_{k} \in X$ :

$$
\begin{aligned}
& \varepsilon_{k}^{1}\left(p_{i j}\right)=p_{i k}+p_{k j}-0.5 ; \\
& \varepsilon_{k}^{2}\left(p_{i j}\right)=p_{k j}-p_{k i}+0.5 ; \\
& \varepsilon_{k}^{3}\left(p_{i j}\right)=p_{i k}-p_{j k}+0.5 .
\end{aligned}
$$

If $P$ is additive consistent, then $\varepsilon_{k}^{1}\left(p_{i j}\right)=\varepsilon_{k}^{2}\left(p_{i j}\right)=\varepsilon_{k}^{3}\left(p_{i j}\right)$ for $i, j, k \in\{1, \ldots, n\}$. Unfortunately, user defined FPRs are not always additive consistent. In this case it is still possible to use equation (2) to identify missing values that are as consistent as possible with the existing ones by mediating the estimates over any defined intermediate alternative as follows:

$$
\varepsilon\left(p_{i j}\right)=\frac{\sum_{l=1}^{3} \sum_{k \in K_{i j}^{l}} \varepsilon_{k}^{l}\left(p_{i j}\right)}{\sum_{l=1}^{3}\left|K_{i j}^{l}\right|}
$$

where the sets $K_{i j}^{l}$ for $l \in\{1,2,3\}$ include the indexes of the defined intermediate alternatives, useful for each estimator of $p_{i j}$. The generation of missing values through equations (2)-(3) is done in several iterations. In each iteration new values are computed based on those previously known and added to the FPR.

Once each expert has expressed her preferences on alternatives of $X$ and missing FPRs values, if any, have been estimated through equations (2)-(3), $m$ individual FPRs are available. A first step needed to reach a final decision is to aggregate available individual FPRs into a collective FPR using some aggregation operator. Several operators have been proposed for this purpose by different researchers like the weighted arithmetic and geometric mean, the min operator and the ordered weighted average $[30,31,32]$.

Once the collective FPR $P$ is obtained, the alternatives must be rated associating a degree of preference $\phi\left(x_{i}\right)$ to each $x_{i} \in X$ basing on $P$. Also in this case several measures exist like the dominance and non- 
dominance degree [33] as well as their quantifier guided versions [7]. Among the simples measures there is the Net Flow proposed in [23] as follows:

$$
\phi\left(x_{i}\right)=\sum_{j=1, j \neq i}^{n} p_{i j}-\sum_{j=1, j \neq i}^{n} p_{j i}
$$

where the first summation is the leaving flow i.e. the total degree of preference of $x_{i}$ over all the other alternatives and the last summation is the entering flow i.e. the total degree of preference of all the other alternatives over $x_{i}$. After having rated the available alternatives with one (or a combination) of the described measures, the one with the highest degree of preference is the solution of the GDM problem.

\section{The Fuzzy Ranking Model}

Given a set of alternatives $X=\left\{x_{1}, \ldots, x_{n}\right\}$, a fuzzy ranking on $X$ can be defined as a sequence $R=$ $\left(\begin{array}{llll}x_{\sigma(1)} s_{1} x_{\sigma(2)} & \ldots & x_{\sigma(k-1)} s_{k-1} x_{\sigma(k)}\end{array}\right)$ with $k \leq n$. Terms in odd positions in the sequence represent a subset of the alternatives, while $\sigma:\{1, \ldots n\} \rightarrow\{1, \ldots k\}$ is a $k$-permutation function. Terms in even positions (separators) belong to the set of symbols $S=\{\gg,>, \geq, \approx\}$ and define a degree of preference between subsequent terms (with $\gg$ meaning "is much better than", $>$ "is better than", $\geq$ "is a little better than" and $\approx$ "is similar to"). Each alternative appears at most once in the ranking so cycles are not allowed although partial rankings are admitted.

Example 1. The fuzzy ranking $R=\left(x_{4} \gg x_{5} \approx x_{2} \geq x_{3}>x_{1}\right)$ defined on $X=\left\{x_{1}, \ldots, x_{5}\right\}$ states that, according to expert's opinion, the fourth alternative is much better than the fifth one that, in turn, is similar to the second one, while both are a little better than the third one that, in turn, is better than the first one.

If we look at Example 1, it becomes clear that, by relying on standard ordinal rankings, it would have been impossible for the same expert to specify her belief so thoroughly. In fact, the ordinal ranking $x_{4}>$ $x_{5}>x_{2}>x_{3}>x_{1}$ that can be extracted from $R$ and can be summarized with the ordering array $O=$ $(5,3,4,1,2)$, has a deeply different semantics: ties are not allowed so the equivalent alternatives $x_{5}$ and $x_{2}$ are artificially ordered while the preference gaps between $x_{4}$ and $x_{5}, x_{2}$ and $x_{3}, x_{3}$ and $x_{1}$ seems comparable in $O$ while they are very different in expert's belief, as expressed in $R$.

Figure 1 graphically illustrates the interpretation of the expert's belief captured by the fuzzy ranking $R$ of Example 1 and by the extracted ordinal ranking $O$. As it can be seen, fuzzy rankings offer more tools to highlight differences between alternatives. Inspired by studies on the use of linguistic labels in GDM $[34,35,36]$, the cardinality of $S$ (i.e. the number of available symbols) has been chosen small enough so as not to impose useless precision to the experts and rich enough to allow a discrimination of the relative performance of the alternatives. On the other hand, the possibility to compose fuzzy rankings by chaining alternatives and symbols, allows to indirectly express a wide variety of preference levels.

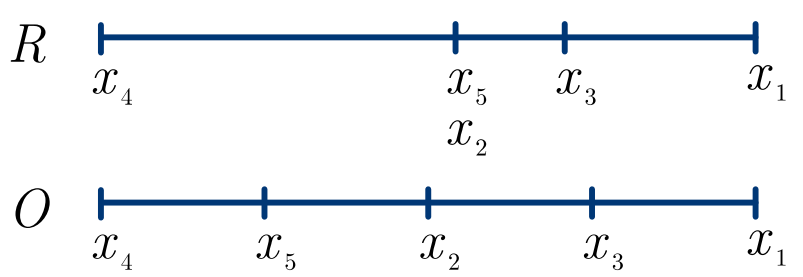

Figure 1. Interpretation of the fuzzy ranking $R$ coming from Example 1 and of the extracted ordinal ranking $O$ 


\section{From Fuzzy Ranking to FPR}

From a fuzzy ranking $R=\left(\begin{array}{lll}x_{\sigma(1)} s_{1} x_{\sigma(2)} \ldots x_{\sigma(k-1)} s_{k-1} x_{\sigma(k)}\end{array}\right)$ it is possible to generate the corresponding FPR $P=\left(p_{i j}\right)$ in several ways. A first approach [15] consists in associating a predefined preference degree $d(s)$ to each symbol $s \in S$ and obtain FPR elements as:

- $p_{\sigma(i) \sigma(i+1)}=d\left(s_{i}\right) \forall i \in\{1, \ldots, k-1\}$;

- $p_{\sigma(i+1) \sigma(i)}=1-d\left(s_{i}\right) \forall i \in\{1, \ldots, k-1\}$;

- $p_{\sigma(i) \sigma(i)}=0.5 \forall i\{1, \ldots, k\}$;

where the first statement transforms the degrees of preference embedded in $R$ in values of $P$, while the second and third statements are aimed at ensuring the reciprocity of $P$ according to the definition given in section 2. A feasible set of values for the function $d(s)$ is shown in Table 1 (second column).

\begin{tabular}{ccc}
\hline \hline Symbol & Preference degree $d(s)$ & Relative strength $|s|$ \\
\hline$\gg$ & 0.85 & 2 \\
$>$ & 0.65 & 1 \\
$\geq$ & 0.58 & 0.5 \\
$\approx$ & 0.50 & 0 \\
\hline \hline
\end{tabular}

Table 1. Feasible values for the preference degree and the relative strength associated to ranking string symbols

It should be noted that, by applying equations (5) on a fuzzy ranking $R$, only $3 k-2$ elements of $P$ can be defined. Even in the case that $R$ involves all available alternatives, (i.e. when $k=n$ ), a number of $n^{2}-3 n+2$ elements of $P$ remain undefined and have to be estimated as explained in section 2 . Moreover, the generated FPR, even when completed in this way, is not guaranteed to be additive consistent.

Example 2. If $R=\left(x_{4} \gg x_{5} \approx x_{2} \geq x_{3}>x_{1}\right)$ is a fuzzy ranking on the set $X=\left\{x_{1}, \ldots, x_{5}\right\}$, the following FPR is generated according to equation (5) using preferences degree values coming from Table 1.

$$
P=\left(\begin{array}{ccccc}
0.5 & - & 0.35 & - & - \\
- & 0.5 & 0.58 & - & 0.5 \\
0.65 & 0.42 & 0.5 & - & - \\
- & - & - & 0.5 & 0.85 \\
- & 0.5 & - & 0.15 & 0.5
\end{array}\right)
$$

where the symbol - indicates an undefined cell. Applying equations (2)-(3) on P we obtain the missing values as follows:

$$
\mathrm{P}=\left(\begin{array}{ccccc}
0.5 & 0.27 & 0.35 & 0 & 0.27 \\
0.73 & 0.5 & 0.58 & 0.15 & 0.5 \\
0.65 & 0.42 & 0.5 & 0.07 & 0.42 \\
1 & 0.85 & 0.93 & 0.5 & 0.85 \\
0.73 & 0.5 & 0.58 & 0.15 & 0.5
\end{array}\right)
$$

A second approach for generating a FPR from a fuzzy ranking is through a transformation function [16]. A relative strength $|\mathrm{s}|$ is associated to each symbol $s \in S$ and, given a fuzzy ranking $R$, a fractional $\operatorname{rank} r\left(x_{i}\right)$ is associated to each alternative so that: 


$$
r\left(x_{\sigma(i)}\right)=1+\sum_{j=1}^{i-1}\left|s_{j}\right|
$$

for $i \in\{1, \ldots, k\}$ and $r\left(x_{i}\right)$ is undefined if $\sigma(i)$ is undefined i.e. if $x_{i}$ does not appear in $R$.

Relative strength values should be selected so that each symbol doubles the strength of the next one. A feasible set of values is shown in Table 1 (third column). By only using the symbol $>$, the fuzzy ranking becomes an ordinal one and equation (6) generates an ordering array as defined in section 2 . The use of the symbols $\gg$ or $\geq$ in place of $>$, respectively doubles or halves the distance of the preceding and subsequent terms in the ranking while the use of $\approx$ means that the preceding and subsequent terms have the same rank. Then, for any pair of alternatives $x_{i}$ and $x_{j}$ appearing in $R$, an element of $P$ can be defined as follows:

$$
p_{i j}=\frac{1}{2}\left(1+\frac{r\left(x_{j}\right)-r\left(x_{i}\right)}{r \max -1}\right)
$$

where $r \max =r\left(x_{\sigma(k)}\right)$ is the maximum rank. The special case $r \max =1$, arising when an expert considers all alternatives as equivalent i.e. when she sets $R=\left(x_{1} \approx \cdots \approx x_{k}\right)$, is handled by directly setting $p_{i j}=0.5$ for any $x_{i}$ and $x_{j}$ appearing in $R$.

Differently from the first approach, by applying equation (7) it is possible to directly define $k^{2}$ elements of the corresponding FPR. When $R$ involves all alternatives, (i.e. when $k=n$ ), the generated FPR presents no undefined elements. In [16] it has been demonstrated that the additive consistency property is verified for all the defined elements a FPR generated from a fuzzy ranking according to equations (6)(7).

Example 3. Let $R=\left(x_{4} \gg x_{5} \approx x_{2} \geq x_{3}>x_{1}\right)$ be the fuzzy ranking of the previous example. Using relative strength values coming from Table 1 in (6), we obtain the following fractional ranks: $r\left(x_{1}\right)=$ 4.5, $r\left(x_{2}\right)=3, r\left(x_{3}\right)=3.5, r\left(x_{4}\right)=1, r\left(x_{5}\right)=3$. Then, according to equation (7), it is possible to generate the corresponding FPR as follows:

$$
P=\left(\begin{array}{ccccc}
0.5 & 0.29 & 0.36 & 0 & 0.29 \\
0.71 & 0.5 & 0.57 & 0.21 & 0.5 \\
0.64 & 0.42 & 0.5 & 0.14 & 0.43 \\
1 & 0.79 & 0.86 & 0.5 & 0.79 \\
0.71 & 0.5 & 0.57 & 0.21 & 0.5
\end{array}\right)
$$

Differently from the previous example, in Example 3 there is no need to complete the FPR with techniques coming from section 2. Moreover, the resulting FPR is additive consistent.

\section{From FPR to Fuzzy Ranking}

In some cases it can be useful to translate the preferences expressed with a FPR $P$ back to a fuzzy ranking. This process can help making manifest and easy to understand experts' defined FPRs or obtaining a meaningful ranking of available alternatives from the collective FPR.

In both cases it is needed to calculate the degree of preference $\phi\left(x_{i}\right)$ of each $x_{i} \in X$ from $P$ as explained in section 2. Then, the fuzzy ranking $R=\left(\begin{array}{lllll}x_{\sigma(1)} s_{1} x_{\sigma(2)} & \ldots & x_{\sigma(n-1)} s_{n-1} & x_{\sigma(n)}\end{array}\right)$ is obtained where $\sigma$ is a permutation function such that $\phi\left(x_{\sigma(i)}\right) \geq \phi\left(x_{\sigma(i+1)}\right)$ and $s_{i} \in S$ for any $i \in\{1, \ldots, n-1\}$. Two approaches can be then adopted (reversing the approaches proposed in section 4 ) to identify the symbols $s_{1}, \ldots, s_{n-1}$.

Given two adjacent alternatives $x_{\sigma(i)}$ and $x_{\sigma(i+1)}$ in $R$, the first approach determines the intermediate symbol $s_{i}$ from the preference value $p_{\sigma(i) \sigma(i+1)}$ of $P$ as follows: 


$$
s_{i}= \begin{cases}\approx & \text { if } p_{\sigma(i) \sigma(i+1)}<0.54 \\ \geq & \text { if } 0.54 \leq p_{\sigma(i) \sigma(i+1)}<0.62 \\ > & \text { if } 0.62 \leq p_{\sigma(i) \sigma(i+1)}<0.75 \\ \gg \quad \text { if } p_{\sigma(i) \sigma(i+1)} \geq 0.75\end{cases}
$$

for any $i \in\{1, \ldots, n-1\}$, where the threshold values $0.54,0.62$ and 0.75 are obtained by averaging each pair of subsequent preference degree values from Table 1. This approach is feasible when the FPR to be translated respects additive transitivity property i.e. when every FPR value is consistent to the others. Otherwise, it may happen that one or more non-coherent values are selected and then incongruent ranking symbols generated.

Example 4. From the additive consistent FPR P resulting from Example 3 it is possible to generate the degree of preference of each alternative in terms of Net Flow according to equation (4) as follows: $\phi_{N F}\left(x_{1}\right)=-2.14 ; \phi_{N F}\left(x_{2}\right)=0 ; \phi_{N F}\left(x_{3}\right)=-0.71 ; \phi_{N F}\left(x_{4}\right)=2.86 ; \phi_{N F}\left(x_{5}\right)=0$. By ordering alternatives based on these values, it is possible to obtain the ordinal ranking: $x_{4}>x_{2}>x_{5}>x_{3}>$ $x_{1}$. The corresponding fuzzy ranking and the related separators can be obtained from equation (8) based on the FPR values: $p_{4,2}=0.79 ; p_{2,5}=0.5 ; p_{5,3}=0.57 ; p_{3,1}=0.64$ as follows: $R=\left(x_{4} \gg x_{2} \approx\right.$ $\left.x_{5} \geq x_{3}>x_{1}\right)$.

Given two adjacent alternatives $x_{\sigma(i)}$ and $x_{\sigma(i+1)}$ in a fuzzy ranking $R$, the second approach determines the intermediate symbol $s_{i}$ from the degrees of preference $\phi\left(x_{\sigma(i)}\right)$ and $\phi\left(x_{\sigma(i+1)}\right)$ associated to the alternatives according to methods described in section 2 :

$$
s_{i}=\left\{\begin{array}{l}
\approx \quad \text { if } \phi\left(x_{\sigma(i+1)}\right)-\phi\left(x_{\sigma(i)}\right)<0.25 \cdot \delta \\
\geq \quad \text { if } 0.25 \cdot \delta \leq \phi\left(x_{\sigma(i+1)}\right)-\phi\left(x_{\sigma(i)}\right)<0.75 \cdot \delta \\
>\quad \text { if } 0.75 \cdot \delta \leq \phi\left(x_{\sigma(i+1)}\right)-\phi\left(x_{\sigma(i)}\right)<1.5 \cdot \delta \\
\gg \quad \text { if }\left(x_{\sigma(i+1)}\right)-\phi\left(x_{\sigma(i)}\right) \geq 1.5 \cdot \delta
\end{array}\right.
$$

where $\delta$ is the average difference between the degrees of preference of two subsequent alternatives in the ranking:

$$
\delta=\frac{1}{n-1} \sum_{i=1}^{n-1}\left(\phi\left(x_{\sigma(i+1)}\right)-\phi\left(x_{\sigma(i)}\right)\right)
$$

and the threshold values $0.25,0.75,0.75$ are obtained by averaging each pair of subsequent relative strength values from Table 1.

Being based only on preference degrees associated to each alternative, the second approach is insensible to the consistency of the original FPR. Moreover, any transformations introduced in the calculation of such degrees of preference according to the methods described in section 2, is considered in the selection of the ranking symbols too.

Example 5. From the FPR resulting from Example 3, after having generated the degree of preference of each alternative in terms of Net Flow, as seen in Example 4, the resulting ordinal ranking is: $x_{4}>$ $x_{2}>x_{5}>x_{3}>x_{1}$. By applying equation (10) on such degrees of preferences we obtain $\delta=1.25$. Basing on equation (9) we can then obtain the fuzzy ranking of available alternatives as: $R=\left(x_{4} \gg\right.$ $\left.x_{2} \approx x_{5} \geq x_{3}>x_{1}\right)$.

\section{Partial and Multiple Fuzzy Rankings}

As specified in section 3, each alternative appears at most once in a fuzzy ranking so partial rankings i.e. rankings involving only $k$ alternatives with $k<n$, are admitted. The exclusion of one or more 
alternatives from a fuzzy ranking means that the expert who defined the ranking is unable to evaluate such alternatives or she considers them incomparable to the others.

In such cases, the transformation methods defined in section 4 produce incomplete FPRs. In particular, if $R$ is a partial fuzzy ranking on the set $X$ and $P=\left(p_{i j}\right)$ is the corresponding FPR obtained with equations (5) or (6)-(7), for any $x_{i} \in X$ not included in $R$, the corresponding elements $p_{i j}$ and $p_{j i}$ remain undefined for any $j \in\{1, \ldots, n\}$. Unfortunately, in this case, missing values cannot be estimated through equations (2)-(3) given that no estimators exist for missing values. In [37], the authors refer to this case as an ignorance situation and suggest to initialize missing values with some seed values that are subsequently refined through an iterative process based on equations (2)-(3) to make them as consistent as possible with the existing values.

Example 6. Let $R=\left(x_{4} \gg x_{5} \approx x_{2}>x_{1}\right)$ be a partial fuzzy ranking on $X=\left\{x_{1}, \ldots, x_{5}\right\}$, using equation (6) with relative strength values coming from Table 1, we obtain the fractional rank of each alternative involved in $R$ as: $r\left(x_{1}\right)=4, r\left(x_{2}\right)=3, r\left(x_{4}\right)=1, r\left(x_{5}\right)=3$. The fractional rank of $x_{3}$ is undefined given that it does not appear in R. According to equation (7), it is then possible to generate the corresponding FPR as follows:

$$
P=\left(\begin{array}{ccccc}
0.5 & 0.33 & - & 0 & 0.33 \\
0.67 & 0.5 & - & 0.17 & 0.5 \\
- & - & - & - & - \\
1 & 0.83 & - & 0.5 & 0.83 \\
0.67 & 0.5 & - & 0.17 & 0.5
\end{array}\right)
$$

The third row and the third column of $P$ are undefined because no information has been provided on $x_{3}$. To complete $P$ it is possible to inject seed values coming from other experts or similar alternatives according to [37]. The simpler (and rougher) method is to set undefined preferences to 0.5 assuming the indifference between $x_{3}$ and any other alternative and then iterate equations (2)-(3) until convergence obtaining the following updated version of $P$ :

$$
P=\left(\begin{array}{ccccc}
0.5 & 0.33 & 0.33 & 0 & 0.33 \\
0.67 & 0.5 & 0.47 & 0.17 & 0.5 \\
0.67 & 0.53 & 0.5 & 0.27 & 0.53 \\
1 & 0.83 & 0.73 & 0.5 & 0.83 \\
0.67 & 0.5 & 0.47 & 0.17 & 0.5
\end{array}\right)
$$

As anticipated in section 3, experts may be allowed to provide multiple fuzzy rankings: sets of partial fuzzy rankings $R_{1}, \ldots, R_{l}$ interesting disjoint subsets of $X$ i.e. so that if an alternative $x_{i} \in X$ appears in a component fuzzy ranking $R_{j}$ with $j \in\{1, \ldots, l\}$, then $x_{i}$ does not appear in any other component ranking $R_{k}$ with $k \in\{1, \ldots, l\} \backslash\{j\}$. The use of multiple fuzzy rankings allows experts to deal with subsets of alternatives they consider as mutually incomparable.

To simplify the notation we can represent a multiple fuzzy ranking within a single sequence $R=$

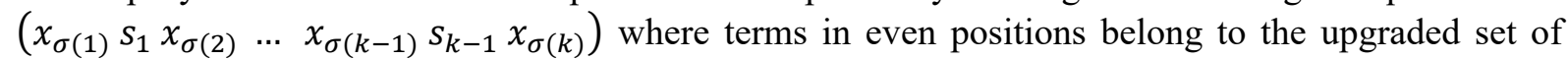
symbols $S \cup\{\Lambda\}$. The additional symbol $\wedge$ is used to interlock the component rankings $R_{1}, \ldots, R_{l}$ interesting disjoint subsets of $X$. Also in this case each alternative appears at most once in the ranking although partial rankings are admitted.

Example 7. The fuzzy ranking $R=\left(x_{4} \gg x_{1} \wedge x_{2} \geq x_{3}>x_{5}\right)$ defined on $X=\left\{x_{1}, \ldots, x_{5}\right\}$ states that the fourth alternative is much better than the fifth one and that the second one is a little better than the third one that, in turn, is better than the first one. Moreover it manifests the expert's inability to compare alternatives coming from the subset $\left\{x_{1}, x_{4}\right\}$ with alternatives coming from $\left\{x_{2}, x_{3}, x_{5}\right\}$.

To obtain a FPR $P$ from a multiple fuzzy ranking $R$ it is enough to iterate equations (5) or (6)-(7) on any component ranking $R_{1}, \ldots, R_{l}$ of $R$ and merge the obtained FPRs $P_{1}, \ldots, P_{l}$. Being $R_{1}, \ldots, R_{l}$ partial fuzzy rankings interesting disjoint subsets of $X$, for any pair of alternatives $x_{i}, x_{j} \in X$ there exist at most one 
FPR $P_{k}=\left(p_{i j}^{k}\right)$ with $k \in\{1, \ldots, l\}$ so that $p_{i j}^{k}$ is defined. For this reason, any element $p_{i j}$ of the overall FPR $P$ can be obtained from the elements of $P_{1}, \ldots, P_{l}$ as follows:

$$
p_{i j}=p_{i j}^{k}: k \in\{1, \ldots, l\}, p_{i j}^{k} \text { is defined. }
$$

When for some $i, j \in\{1, \ldots, n\}, p_{i j}^{k}$ is undefined for any $k \in\{1, \ldots, l\}$ then $p_{i j}$ remains undefined too. This case happens when $x_{i}$ and $x_{j}$ only appear in different component rankings of $R$ or when either $x_{i}$ or $x_{j}$ do not appear at all in any component ranking of $R$. In particular, the latter case happens when the multiple ranking is also a partial ranking.

Example 8. The multiple fuzzy ranking $R=\left(x_{4} \gg x_{1} \wedge x_{2} \geq x_{3}>x_{5}\right)$ coming from the previous example can be split in the two component rankings $R_{1}=\left(x_{4} \gg x_{1}\right)$ and $R_{2}=\left(x_{2} \geq x_{3}>x_{5}\right)$. Applying equation (6) we obtain that $r\left(x_{1}\right)=3, r\left(x_{2}\right)=1$ from $R_{1}$ and $r\left(x_{2}\right)=1, r\left(x_{3}\right)=1.5$, $r\left(x_{5}\right)=2.5$ from $R_{2}$. Applying equation (7) on such fractional ranks we then obtain the following FPRs:

$$
P_{1}=\left(\begin{array}{ccccc}
0.5 & - & - & 0 & - \\
- & - & - & - & - \\
- & - & - & - & - \\
1 & - & - & 0.5 & - \\
- & - & - & - & -
\end{array}\right) ; P_{2}=\left(\begin{array}{ccccc}
- & - & - & - & - \\
- & 0.5 & 0.67 & - & 1 \\
- & 0.33 & 0.5 & - & 0.83 \\
- & - & - & - & - \\
- & 0 & 0.17 & - & 0.5
\end{array}\right) .
$$

Merging $P_{1}$ and $P_{2}$ through equation (11) the following FPR is obtained:

$$
P=\left(\begin{array}{ccccc}
0.5 & - & - & 0 & - \\
- & 0.5 & 0.67 & - & 1 \\
- & 0.33 & 0.5 & - & 0.83 \\
1 & - & - & 0.5 & - \\
- & 0 & 0.17 & - & 0.5
\end{array}\right)
$$

As it can be seen, preference values between alternatives from $\left\{x_{1}, x_{4}\right\}$ (that are referenced in $R_{1}$ ) and alternatives from $\left\{x_{2}, x_{3}, x_{5}\right\}$ (that are referenced in $R_{2}$ ) remain undefined. As for Example 6 it is possible to estimate missing values with methods proposed in [37].

\section{Similarity Between Fuzzy Rankings}

In GDM problems, each expert $e_{i} \in E$ defines an individual fuzzy ranking $R_{i}$ on the same set $X=$ $\left\{x_{1}, \ldots, x_{n}\right\}$. To assess the level of agreement between experts, it is useful to define similarity measures between fuzzy rankings. A possible approach for that is to extend to fuzzy rankings existing similarity measures defined on ordinal rankings like Kendall's and Spearman's rank correlation coefficients.

Let $O_{i}$ and $O_{j}$ be two ordinal rankings on $X$, the Kendall's rank correlation coefficient $[38,39]$ is defined as:

$$
\tau\left(O_{i}, O_{j}\right)=\frac{2\left(c_{i j}-d_{i j}\right)}{n(n-1)}
$$

where $c_{i j}$ is the number of concordant pairs and $d_{i j}$ the number of discordant pairs between $O_{i}$ and $O_{j}$. A concordant pair is pair of alternatives of $X$ which have the same order in the two rankings while a discordant pair is a pair of alternatives which have the opposite order in the two rankings.

To extend the Kendall's rank correlation coefficient to fuzzy rankings it is needed to redefine $c_{i j}$ and $d_{i j}$ based on the notion of fractional rank given in section 4 so taking ties and partial rankings into account. If $r_{i}\left(x_{k}\right)$ denotes the fractional rank of an alternative $x_{k} \in X$ in a fuzzy ranking $R_{i}$ and $\delta r_{k l}^{i}=$ $r_{i}\left(x_{k}\right)-r_{i}\left(x_{l}\right)$ for $x_{k}, x_{l} \in X$, we can say that $\left(x_{k}, x_{l}\right)$ is a concordant pair between two ranking $R_{i}$ and 
$R_{j}$ on $X$ if both alternatives appear in both rankings and the condition $\delta r_{k l}^{i} \cdot \delta r_{k l}^{j}>0$ or $\delta r_{k l}^{i}=\delta r_{k l}^{j}=0$ is verified (i.e. $\delta r_{k l}^{i}$ and $\delta r_{k l}^{j}$ are both positive, both negative or both 0 ). Conversely, $\left(x_{k}, x_{l}\right)$ is a discordant pair if both alternatives appear in $R_{i}$ and $R_{j}$ but the preceding condition is not met (i.e. $\delta r_{k l}^{i}$ and $\delta r_{k l}^{j}$ are one positive and the other negative or one equal to 0 and the other different from 0 ).

Based on $c_{i j}$ and $d_{i j}$ we can define the Kendall's correlation coefficient for fuzzy rankings as:

$$
\tau\left(R_{i}, R_{j}\right)=\frac{2\left(c_{i j}-d_{i j}\right)}{\operatorname{kmax}_{i j}\left(\operatorname{kmax}_{i j}-1\right)}
$$

where $\operatorname{kmax}_{i j}=\max \left(k_{i}, k_{j}\right)$ while $k_{i}$ and $k_{j}$ are the number of alternatives involved, in turn, in $R_{i}$ and $R_{j}\left(\right.$ with $\left.k_{i}, k_{j} \leq n\right)$.

Example 9. Let $R_{1}, \ldots, R_{5}$ be the fuzzy rankings shown in the first column of Table 2, defined on $X=$ $\left\{x_{1}, \ldots, x_{5}\right\}$. Using equation (6) we obtain, for each ranking $R_{i}$, the fractional ranks reported in columns 2-6 of the same table. Then, exploiting the definition of concordant and discordant pairs, we obtain: $c_{1,2}=6, c_{1,3}=10, c_{1,4}=6, c_{1,5}=1, d_{1,2}=4, d_{1,3}=0, d_{1,4}=0, d_{1,5}=9$. By considering that $\operatorname{kmax}_{i j}=5$ for any $i, j \in\{1, \ldots, 5\}$ we obtain from equation (13): $\tau\left(R_{1}, R_{2}\right)=0.2$ (weak positive correlation), $\tau\left(R_{1}, R_{3}\right)=1$ (equivalence), $\tau\left(R_{1}, R_{4}\right)=0.6$ (moderate positive correlation), $\tau\left(R_{1}, R_{5}\right)=-0.8$ (strong negative correlation).

\begin{tabular}{lccccc}
\hline \hline Ranking & $r_{i}\left(x_{1}\right)$ & $r_{i}\left(x_{2}\right)$ & $r_{i}\left(x_{3}\right)$ & $r_{i}\left(x_{4}\right)$ & $r_{i}\left(x_{5}\right)$ \\
\hline$R_{1}=\left(x_{4} \gg x_{5} \approx x_{2} \geq x_{3}>x_{1}\right)$ & 4.5 & 3 & 3.5 & 1 & 3 \\
$R_{2}=\left(x_{5}>x_{4} \geq x_{3} \geq x_{2} \approx x_{1}\right)$ & 3 & 3 & 2.5 & 2 & 1 \\
$R_{3}=\left(x_{4} \geq x_{5} \approx x_{2} \gg x_{3} \geq x_{1}\right)$ & 4 & 1.5 & 3.5 & 1 & 1.5 \\
$R_{4}=\left(x_{4} \gg x_{5} \approx x_{2}>x_{1}\right)$ & 4 & 3 & - & 1 & 3 \\
$R_{5}=\left(x_{3} \approx x_{1} \geq x_{5} \gg x_{4} \geq x_{5}\right)$ & 1 & 1.5 & 1 & 3.5 & 4 \\
\hline \hline
\end{tabular}

Table 2. Five sample fuzzy rankings and the fractional rank of each involved alternative

A limit of the Kendall's correlation coefficient is that it considers only the position of alternatives in the ranking disregarding the preference gaps quantified by the separators. In addition, it can bring some pragmatic issues in case the rankings are not precise as outlined in [40]. In fact, in Example 9, $R_{1}$ and $R_{3}$ are considered as equivalent even if, by looking at the separators used, we can see that the experts' beliefs captured by the two rankings are quite different. In fact the preference gap between $x_{4}$ and $x_{5}$ is wide in $R_{1}$ and thin in $R_{3}$ while the preference gap between $x_{2}$ and $x_{3}$ is thin in $R_{1}$ and wide in $R_{3}$ [16]. In [41] the Spearman's correlation coefficient is introduced as a measure of consensus in GDM problems modeled with FPRs. Following that results, with the aim of taking fuzzy separators into account when computing the similarity between fuzzy rankings, we introduce the Spearman's correlation coefficient for fuzzy rankings as an extension of the homonymous correlation coefficient for ordinal rankings defined in [42] as follows:

$$
\rho\left(R_{i}, R_{j}\right)=\frac{\sum_{k=1}^{n}\left(r_{i}\left(x_{k}\right)-\bar{r}_{l}\right)\left(r_{j}\left(x_{k}\right)-\bar{r}_{j}\right)}{\sqrt{\sum_{k=1}^{n}\left(r_{i}\left(x_{k}\right)-\bar{r}_{l}\right)^{2}} \sqrt{\sum_{k=1}^{n}\left(r_{j}\left(x_{k}\right)-\bar{r}_{j}\right)^{2}}}
$$

where $\bar{r}_{l}=\frac{1}{n} \sum_{k=1}^{n} r_{i}\left(x_{k}\right)$ is the average fractional rank extracted from $R_{i}$ and $\bar{r}_{j}$ is the average fractional rank extracted from $R^{j}$ in the same way. 
Example 10. Let $R_{1}, \ldots, R_{5}$ be the fuzzy rankings defined in Example 9 and summarized in Table 2 with their fractional ranks. By applying equation (14) we obtain the following values for the Spearman's correlation coefficient: $\rho\left(R_{1}, R_{2}\right)=0.41$ (moderate positive correlation), $\rho\left(R_{1}, R_{3}\right)=0.83$ (strong positive correlation), $\rho\left(R_{1}, R_{4}\right)=0.97$ (very strong positive correlation), $\rho\left(R_{1}, R_{5}\right)=-0.68$ (moderate negative correlation).

By looking at the results of Example 10, it can be seen that $R_{1}$ and $R_{3}$ are only strongly correlated according to the Spearman's coefficient rather than equivalent as in the previous case. This happens because the Spearman's coefficient, being based on differences between fractional ranks, also takes into account the preference gaps quantified by the separators that are used within the fuzzy ranking.

\section{Comparison with Related Works}

To the best of our knowledge, the concept of fuzzy ranking is quite new. We have presented a first definition of this model in [15] with the name of ranking string together with a translation method to FPR (the first approach seen in section 4). In [16], we have defined a new, more performing translation method to FPR (the second approach seen in section 4). In the present work, after having formalized and extended the model outlined in such previous papers, we have included useful special cases (like partial and multiple fuzzy rankings), defined conversion functions from FPR backward to fuzzy ranking as well as similarity measures.

An alternative formulation of the fuzzy ranking concept has been recently proposed in [43] as a generalization of crisp rankings. While in a crisp ranking each object is assigned just one position, in the fuzzy ranking model defined in [43], the same object may be assigned to many positions with different degrees of membership. So, to characterize it, an $n \times n$ ordering matrix $R$ is used whose generic element $r_{i j} \in[0,1]$ denotes the membership degree of the $i$-th object to the $j$-th position and $\sum_{i=1}^{n} r_{i j}=\sum_{j=1}^{n} r_{i j}=1$ for all $i, j \in\{1, \ldots, n\}$.

The main difference with respect our model resides in the way the ranking concept is fuzzyfied. Instead of allowing the same object belong to multiple positions, in fact, our model allows to extend or contract the gap between subsequent positions to reinforce or weaken the ordering relation. As well as being more useful to support preferences expression in GDM, our approach also allows the use of a more compact and user-friendly notation for rankings definition. The definition of an ordering matrix, like that needed for the model described in [43] is in fact quite difficult and comparable to the direct definition of a FPR, nullifying in this way any advantage carried out by the adoption of an alternative model.

In $[44,45]$ the Linguistic Preference Relations (LPR) have been defined as an alternative to the FPR. In LPR, the relative preference of each alternative with respect to any other is expressed with a linguistic term (that is subsequently converted into a fuzzy number) rather than with a membership degree in $[0,1]$. A LPR can be so represented with an $n \times n$ matrix $P=\left(p_{i j}\right)$ where each element $p_{i j}$ states the linguistically assessed preference degree of the alternative $x_{i}$ over $x_{j}$.

Similarly to LPRs, fuzzy rankings allow to specify fuzzy statements about pairs of alternatives, differently from LPRs (where a linguistic term must be chosen for every pair of alternatives), in fuzzy rankings a fuzzy statement is specified only for a subset of all possible pairs i.e. only for alternatives that are adjacent in the ranking. On one hand, this allows fuzzy rankings to adopt a more compact and meaningful notation; on the other hand, it is possible to easily infer missing preferences by avoiding inconsistencies. Moreover, while fuzzy rankings can be transformed in FPRs and processed with standard GDM methods and tools, LPRs need specific fuzzy extensions of such methods and tools.

A topic quite related to fuzzy rankings is that of fuzzy numbers ranking. How to rank fuzzy numbers is an important problem in GDM, and is particularly felt when experts use fuzzy estimates (maybe expressed in form of linguistic terms) to specify their preferences. According to [46], more than 30 fuzzy ranking indices have been proposed since 1976 for this purpose. By directly using fuzzy rankings instead of fuzzy estimates to specify preferences can be considered as a convenient and user-friendly method to overcome the fuzzy numbers ranking issue. 
In $[47,48]$ the FSQL language has been proposed to handle fuzzy information within databases. In order to perform queries involving fuzzy quantities, such language introduces several fuzzy comparators like: $F=$ (fuzzy equal than), $F<>$ (fuzzy different to), $F>$ (fuzzy greater than), $F>=$ (fuzzy greater or equal than), $F<$ (fuzzy less than), $F<=$ (fuzzy less or equal than), $F \gg$ (fuzzy much greater than), $F \ll$ (fuzzy much less than) where each comparator is associated to an algorithm able to compare fuzzy numbers as those used for ranking.

As it can be noted, there is a substantial similarity between symbols used by fuzzy comparators and those adopted by fuzzy rankings. Nevertheless, fuzzy rankings use such symbols to state fuzzy relations about crisp objects rather than to assess if a crisp relation exists between fuzzy quantities. For this reason, even if syntactically similar, the semantics under these symbols is very different.

\section{Final Remarks}

This paper defines a new approximate preference model for GDM named fuzzy ranking that combines the user-friendliness of the ordinal ranking model with the expressive capability of FPRs. Like FPRs, fuzzy rankings allow experts to focus on two alternatives at a time, in fact meaningful separators must be placed between pair of alternatives. Differently from FPRs it is not needed to assess preference degrees for any pair of available alternatives (resulting in $n^{2}$ comparisons with $n$ alternatives) but just for adjacent alternatives in the defined ranking (resulting in just $n-1$ comparisons).

Fuzzy rankings offer a compact notation that do not impose unnecessary precision in preference definition. In this way it is impossible to introduce inconsistencies in the GDM process while allowing to reason by approximation. The impossibility to evaluate alternatives is supported with partial fuzzy rankings while the incomparability between alternatives is allowed through multiple fuzzy rankings. To let use standard GDM methods and tools even when preferences are expressed with fuzzy rankings translation functions to and from FPRs are provided as well as similarity measures to assess the convergence of experts' opinions.

Fuzzy rankings have been already experimented even for the expression of ordinal assessment statements within a work aimed at the application of GDM techniques in peer grading [15] and for the collection of expert preferences within a GDM model guided by social influence [16, 49]. The first outcomes regarding their adoption are positive both when supported by results coming from in-silico experiments (like in $[15,16])$ than when such models are applied in real contexts (like in $[50,51])$ and encourage its application in further application contexts $[52,53]$.

\section{Acknowledgments}

The authors would like to acknowledge FEDER financial support from the Project TIN2016-75850-R.

\section{References}

[1] F. J. Cabrerizo, F. Chiclana, R. Al-Hmouz, A. Morfeq, A. S. Balamash and E. Herrera-Viedma, "Fuzzy decision making and consensus," Journal of Intelligent and Fuzzy Systems, vol. 29, no. 3, pp. 1109-1118, 2015.

[2] D. Yu, J. M. Merigó and Y. Xu, "Group Decision Making in Information Systems Security Assessment Using Dual Hesitant Fuzzy Set," International Journal of Intelligent Systems, vol. 31, pp. 786-812, 2016.

[3] W. Pedrycz, P. Ekel and R. Parreiras, Fuzzy Multicriteria Decision-Making: Models, Methods and Applications, John Wiley \& Sons, 2010.

[4] F. Cabrerizo, J. Moreno, I. Pérez and E. Herrera-Viedma, "Analyzing consensus approaches in fuzzy group decision making: advantages and drawbacks.," Soft Computing, vol. 14, no. 5, pp. 451-463, 2010. 
[5] H. Zhang, Y. Dong and E. Herrera-Viedma, "Consensus building for the heterogeneous large-scale GDM with the individual concerns and satisfactions," IEEE Trans. On Fuzzy Systems, $2017 . \quad$ Doi: 10.1109/TFUZZ.2017.2697403.

[6] M. Del Moral, F. Chiclana, J. Tapia and E. Herrera-Viedma, "A Comparative Study on Consensus Measures in Group Decision Making," Int. J. of Intelligent Systems, 2017.

[7] F. Chiclana, F. Herrera and E. Herrera-Viedma, "Integrating three representation models in fuzzy multipurpose decision making based on fuzzy preference relations," Fuzzy Sets and Systems, vol. 97, no. 1, pp. 33-48, 1998.

[8] V. Belton, "Multiple-criteria problem structuring and analysis in a value theory framework," in Multicriteria Decision Making: Advances in MCDM Models, Algorithms, Theory, Kluwer, 1999, pp. 2-29.

[9] R. J. Li, "Fuzzy method in group decision making," Computers \& Mathematics with Applications, vol. 38, no. 1, pp. 91-101, 1999.

[10] Z. Xu and W. Cuiping, "A consistency improving method in the analytic hierarchy process," European Journal of Operational Research, vol. 116, no. 2, pp. 443-449, 1999.

[11] K. Y. Wong and M. E. Spetsakis, "Tracking, Segmentation and Optical Flow," York, Canada, 2003.

[12] F. Seo and M. Sakawa, "Fuzzy multiattribute utility analysis for collective choice," IEEE Transactions on Systems, Man and Cybernetics, vol. 15, no. 1, pp. 45-53, 1985.

[13] W. Liu, Y. Dong, F. Chiclana, F. J. Cabrerizo and E. Herrera-Viedma, "Group decision-making based on heterogeneous preference relations with self-confidence," Fuzzy Optimization and Decision Making, vol. 16, no. 4, p. 429-447, 2017.

[14] M. R. Ureña, F. Chiclana, J. A. Morente-Molinera and E. Herrera-Viedma, "Managing Incomplete Preference Relations in Decision Making: A Review and Future Trends," Information Sciences, vol. 302, no. 1, pp. 14-32, 2015.

[15] N. Capuano, V. Loia and F. Orciuoli, "A Fuzzy Group Decision Making Model for Ordinal Peer Assessment," IEEE Transactions on Learning Technology, vol. 10, no. 2, pp. 247-259, 2017.

[16] N. Capuano, F. Chiclana, H. Fujita, E. Herrera-Viedma and V. Loia, "Fuzzy Group Decision Making with Incomplete Information Guided by Social Influence," IEEE Transaction of Fuzzy Systems, 2017. http://dx.doi.org/10.1109/TFUZZ.2017.2744605.

[17] F. J. Cabrerizo, E. Herrera-Viedma and W. Pedrycz, "A Method based on PSO and Granular Computing of Linguistic Information to Solve Group Decision Making Problems defined in Heterogeneous Contexts," European Journal of Operational Research, vol. 230, no. 3, pp. 624-633, 2013.

[18] J. Wu and F. Chiclana, "A social network analysis trust-consensus based approach to group decision-making problems with interval-valued fuzzy reciprocal preference relations," Knowledge-Based Systems, vol. 59, pp. 97107,2014

[19] Y. Zhai, Z. Xu and H. Liao, "Probabilistic linguistic vector-term set and its application in group decision making with multi-granular linguistic information," Applied Soft Computing, vol. 49, pp. 801-816, 2016.

[20] F. Meng and Q. An, "A new approach for group decision making method with hesitant fuzzy preference relations," Knowledge-Based Systems, vol. 127, pp. 1-15, 2017.

[21] S. Zhang, J. Zhu, X. Liu, Y. Chen and Z. Ma, "Adaptive consensus model with multiplicative linguistic preferences based on fuzzy information granulation," Applied Soft Computing, vol. 60, pp. 30-47, 2017.

[22] S. Destercke and I. Couso, "Ranking of fuzzy intervals seen through the imprecise probabilistic lens," Fuzzy Sets and Systems, vol. 278, pp. 20-39, 2015.

[23] Y. M. Wang and Z. P. Fan, "Fuzzy preference relations: Aggregation and weight determination," Computers \& Industrial Engineering, vol. 53, no. 1, pp. 163-172, 2007.

[24] Atiq-ur-Rehman, E. E. Kerre and S. Ashraf, "Group Decision Making by Using Incomplete Fuzzy Preference Relations Based on T-Consistency and the Order Consistency," International Journal of Intelligent Systems, vol. 30, pp. 120-143, 2014.

[25] J. Ramík, "Incomplete Fuzzy Preference Matrix and Its Application to Ranking of Alternatives," International Journal of Intelligent Systems, vol. 29, pp. 787-806, 2014.

[26] S. Alonso, F. Chiclana, F. Herrera, E. Herrera-Viedma, J. Alcala-Fdez and C. Porcel, "A consistency-based procedure to estimate missing pairwise preference values," International Journal of Intelligent Systems, vol. 23, no. 1, pp. 155-175, 2008. 
[27] E. Herrera-Viedma, F. Chiclana, F. Herrera and S. Alonso, "Group Decision-Making Model with Incomplete Fuzzy Preference Relations Based on Additive Consistency," IEEE Transactions on Systems, Man and Cybernetics, Part B, Cybernetics, vol. 37, no. 1, pp. 176-189, 2007.

[28] F. Chiclana, E. Herrera-Viedma, S. Alonso and F. Herrera, "Cardinal Consistency of Reciprocal Preference Relations: A Characterization of Multiplicative Transitivity," IEEE Transactions on Fuzzy Systems, vol. 17, no. 1, pp. 14-23, 2009.

[29] F. Chiclana, E. Herrera-Viedma, S. Alonso and F. Herrera, "A Note on the Estimation of Missing Pairwise Preference Values: A Uninorm Consistency Based Method," International Journal of Uncertainty, Fuzziness and Knowledge Based Systems, vol. 16, no. 2, pp. 19-32, 2008.

[30] R. R. Yager, "Families of OWA operators," Fuzzy Sets and Systems, vol. 59, no. 2, pp. 125-148, 1993.

[31] H. Liao and Z. Xu, "Extended hesitant fuzzy hybrid weighted aggregation operators and their application in decision making," Soft Computing, vol. 19, no. 9, pp. 2551-2564, 2015.

[32] G. Beliakov, T. Calvo and S. James, "Aggregation Functions for Recommender Systems," in Recommender Systems Handbook, 2015, pp. 777-808.

[33] S. A. Orlovsky, "Decision making with a fuzzy preference relation," Fuzzy Sets and Systems, vol. 1, no. 3, pp. 155-167, 1978.

[34] C. C. Li, Y. Dong, F. Herrera, E. Herrera-Viedma and L. Martínez, "Personalized individual semantics in Computing with Words for supporting linguistic Group Decision Making. An Application on Consensus reaching," Information Fusion, vol. 33, no. 1, pp. 29-40, 2017.

[35] Y. Dong and E. Herrera-Viedma, "Consistency-driven automatic methodology to set interval numerical scales of 2-tuple linguistic term sets and its use in the linguistic GDM with preference relation," IEEE Transactions on Cybernetics, vol. 45, no. 4, pp. 780-792, 2015.

[36] Y. Zhai, Z. Xu and H. Liao, "Probabilistic linguistic vector-term set and its application in group decision making with multi-granular linguistic information," Applied Soft Computing, vol. 49, pp. 801-816, 2016.

[37] S. Alonso, E. Herrera-Viedma, F. Chiclana and F. Herrera, "Individual and Social Strategies to Deal with Ignorance Situations in Multi-Person Decision Making," International Journal of Information Technology and Decision Making, vol. 8, no. 2, pp. 313-333, 2009.

[38] M. Kendall, "A New Measure of Rank Correlation," Biometrika, vol. 30, no. 1-2, pp. 81-89, 1938.

[39] L. A. Goodman and W. H. Kruskal, "Measures of Association for Cross Classifications," Journal of the American Statistical Association, vol. 49, no. 268, pp. 732-764, 1954.

[40] I. Couso, O. Strauss and H. Saulnier, "Kendall's rank correlation on quantized data: An interval-valued approach," Fuzzy Sets and Systems, vol. In press, 2017.

[41] T. Gonzalez-Arteaga, R. De Andres Calle and F. Chiclana, "A new measure of consensus with fuzzy preference relations: The correlation consensus degree," Knowledge-Based Systems, vol. 107, pp. 104-116, 2016.

[42] C. Spearman, "The proof and measurement of association between two things," American Journal of Psychology, vol. 15, pp. 72-101, 1904.

[43] J. Mazurek, "Fuzzy Rankings: Properties and Applications," Cornell University Library - arXiv, 2017.

[44] J. Wu, F. Chiclana and E. Herrera-Viedma, "Trust Based Consensus Model for Social Network in an Incomplete Linguistic Information Context," Applied Soft Computing, vol. 35, pp. 827-839, 2015.

[45] F. Cabrerizo, M. Ureña, W. Pedrycz and E. Herrera-Viedma, "Building consensus in group decision making with an allocation of information granularity," Fuzzy Sets and Systems, vol. 255, pp. 115-127, 2014.

[46] S. Abbasbandy, "Ranking of Fuzzy Numbers, Some Recent and New Formulas," in Proceedings of the Joint International Fuzzy Systems Association World Congress and European Society of Fuzzy Logic and Technology Conference, Lisbon, Portugal, 2009.

[47] J. Galindo, A. Urrutia and M. Piattini, Fuzzy Databases: Modeling, Design and Implementation, IGI Global, 2005.

[48] J. Galindo, "New Characteristics in FSQL, a Fuzzy SQL for Fuzzy Databases," WSEAS Transactions on Information Science and Applications, vol. 2, no. 2, pp. 161-169, 2005.

[49] L. Perez, F. Mata, F. Chiclana, G. Kou and E. Herrera-Viedma, "Modelling influence in group decision making," Soft Computing, vol. 20, no. 4, pp. 1653-1665, 2016.

[50] G. Albano, N. Capuano and A. Pierri, "Adaptive Peer Grading and Formative Assessment," Journal of e-Learning and Knowledge Society, vol. 13, no. 1, pp. 147-161, 2017. 
[51] N. Capuano and F. Orciuoli, "Application of Fuzzy Ordinal Peer Assessment in Formative Evaluation," in Proceedings of the 12th International Conference on P2P, Parallel, Grid, Cloud and Internet Computing (3PGCIC 2017), Barcelona, Spain, 2017.

[52] I. Pérez, F. Cabrerizo and E. Herrera-Viedma, "A Mobile Decision Support System for Dynamic Group Decision Making Problems," IEEE Transactions on Systems, Man and Cybernetics - Part A: Systems and Humans, vol. 40, no. 6, pp. 1244-1256, 2010.

[53] J. Wu, F. Chiclana, H. Fujita and E. Herrera-Viedma, "A visual interaction consensus model for social network group decision making with trust propagation," Knowledge Based-Systems, vol. 122, pp. 39-50, 2017.

[54] S. Alonso, F. J. Cabrerizo, F. Chiclana, F. Herrera and E. Herrera-Viedma, "Group Decision-Making with Incomplete Fuzzy Linguistic Preference Relations," International Journal of Intelligent Systems, vol. 24, no. 2, pp. 201-222, 2009. 